\title{
Site Distribution in Resin Beads as Determined by Confocal Raman Spectroscopy**
}

\author{
Jürgen Kress, ${ }^{[a]}$ Abigail Rose, ${ }^{[a]}$ Jeremy G. Frey, ${ }^{[a]}$ William S. Brocklesby, ${ }^{[b]}$ Mark Ladlow, ${ }^{[c]}$ \\ Geoff W. Mellor, ${ }^{[\mathrm{d}]}$ and Mark Bradley*[a]
}

\begin{abstract}
Scanning confocal Raman spectroscopy was used to study the distribution of reactive sites within a resin bead used for solid-phase synthesis. The distribution of $\mathrm{NH}_{2}$ groups in aminomethylated polystyrene resin (APS) was determined by doping with varying amounts of 4-cyanobenzoic acid. The extent of loading was determined by
\end{abstract}

both elemental analysis and ninhydrin assays. The spatial distribution of the coupled 4-cyanobenzamide within the

Keywords: combinatorial chemistry - confocal microscopy - polymers • Raman spectroscopy • solid-phase synthesis bead was determined to an in-plane resolution of $1 \mu \mathrm{m}$ and depth resolution of about $4 \mu \mathrm{m}$, using the strong Raman $\mathrm{CN}$ stretching vibrational transition at $2230 \mathrm{~cm}^{-1}$. Dry and swollen beads were studied and the distribution was found to be essentially uniform throughout the bead in all cases.

\section{Introduction}

The way in which organic chemistry is being performed is being dramatically influenced by developments in solid-phase supported chemistry. ${ }^{[1 \mathrm{a}-\mathrm{d}]}$ Routes based on the use of scavengers and immobilised reagents are enhancing the impact of traditional solid-phase approaches. ${ }^{[1 e]}$ Pivotal to this effort is the solid support itself and the way in which this influences the efficiency of the desired chemical transformations. However, new solid-phase chemistries cannot simply be taken from existing solution literature without modification, in part due to the additional complexities of the solid support itself, for example changes in media polarity, solvation, steric con-

[a] Prof. Dr. M. Bradley, Dr. J. Kress, A. Rose, Dr. J. G. Frey Department of Chemistry

University of Southampton, Southampton SO17 1BJ (Great Britain) Fax: $(+44) 2380-596766$

E-mail: j.quinn-parsons@soton.ac.uk

[b] Dr. W. S. Brocklesby

Department of Physics and Optoelectronics Research Centre

University of Southampton

Southampton SO17 1BJ (Great Britain)

[c] Dr. M. Ladlow

Chemical \& Analytical Technologies

Glaxo Wellcome Cambridge Chemistry Unit

Lensfield Road, Cambridge CB2 1E (Great Britain)

[d] Dr. G. W. Mellor

GlaxoWellcome Medicines Research Centre

Gunnels Wood Road, Stevenage SG1 2NY (Great Britain)

[**] Editorial note: For a similar study using confocal fluorescent microscopy, see the following paper by J. Rademann, M. Barth, R. Brock, H.-J. Egelhaaf, G. Jung, Chem. Eur. J. 2001, 7, 3884-3889. straints within the bead or changes in the partitioning of compounds between the different environments. Therefore, any investigation that provides a detailed understanding and picture of the properties of common solid-phase synthetic supports is of interest. Although many physical characteristics of those polymeric supports have been published, ${ }^{[2]}$ many important questions remain unanswered, in particular in relation to the distribution and relative accessibility of reactive functionalities within a given bead type. ${ }^{[3]}$

As early as 1980 autoradiography provided evidence for the homogeneous distribution of the functionalities throughout polystyrene (PS) resin beads (1\% cross-linked with divinylbenzene (DVB) $),{ }^{[4]}$ as did scanning secondary ion mass spectrometry some years later. ${ }^{[5]}$ However, a recent paper was at odds with these conclusions. Using conventional fluorescent labelling and optical detection techniques, ${ }^{[6]}$ a dramatic variation in site distribution was observed across conventional gel based PS and TentaGel (TG $)^{[7]}$ resin beads. The same technique was used recently for the investigation of so-called Rasta-resin, a uniform site-distribution was found. ${ }^{[8]}$

\section{Results and Discussion}

Extracting the site distributions from fluorescence experiments on fluorophore loaded beads is not straightforward because of problems associated with quenching and reabsorption of the fluorescence. ${ }^{[9]}$ The small volume of a typical $100 \mu \mathrm{m}$ diameter bead with a loading of 400 pmoles per bead gives a fluorophore concentration within the bead 
that approaches $300 \mathrm{~mm}$. Given the high absorption crosssection of a typical dye, this concentration in the bead would lead to absorption of about $99 \%$ per micron and the bead becomes "optically thick". This results in significant absorption of the incident excitation light as it travels through the bead and the radiation does not uniformly excite all sites. Similarly such high loadings result in significant absorption and re-absorption of the fluorescence and therefore cause an apparent nonlinear response of fluorescence intensity with bead loading. Furthermore, at these concentrations of the fluorophore, even in solution, quenching would be expected. In addition interactions between closely spaced fluorophore molecules can lead to spectral shifts and broadening of the transitions.

We therefore decided to clarify the situation, and obtain greater detail on the distribution and reactivity of sites within the resin bead using high resolution confocal Raman microscopy; a non-fluorescent method. ${ }^{[10,11]}$ In conventional confocal microscopy a pin-hole is used in both the downward and return optical path to restrict both the lateral extent of the image and the depth of focus. This enables true three-dimensional images to be recorded slice by slice through the sample.

The most commonly used gel-type resins in solid-phase synthesis are the commercially available low-cross-linked PS supports (1-2\% DVB, typical loading $\left.1.2 \mathrm{mmolg}^{-1}\right)$ and TentaGel (TG, typical loading $0.23 \mathrm{mmol} \mathrm{g}^{-1}$ ) resin. These beads only become gel-like and synthetically useful after swelling in an appropriate solvent and therefore the studies were undertaken with both dry and swollen beads. The distribution of $\mathrm{NH}_{2}$ groups in aminomethylated polystyrene resins (APS) was therefore determined by doping the resins with varying amounts of 4-cyanobenzoic acid. The extent of loading was determined by both elemental analysis and ninhydrin assay. ${ }^{[12]}$ The cyano group has a strong Raman transition at $2230 \mathrm{~cm}^{-1}$ which is easily resolved amongst the Raman spectrum of the bead polymer. The Raman spectra of the beads were collected with a Renishaw Raman imaging microscope 2000 system. The spectra from the whole bead were collected using the continuous extended grating mode ( $\times 20$ objective, $20 \mathrm{~s}$ exposure, three accumulations, 1800 grooves $\mathrm{mm}^{-1}$, grating with a $20 \mu \mathrm{m}$ slit giving a spectral resolution of $\approx 0.1 \mathrm{~cm}^{-1}$ ).

Aminomethylated polystyrene resin beads (loading $1.23 \mathrm{mmol} \mathrm{g}^{-1}$ ) were loaded with varying amounts of 4-cyanobenzoic acid, obtained by quenching the coupling reaction after different reaction times as described in the Experimental Section. Table 1 gives the loading (established by duplicate quantitative ninhydrin tests ${ }^{[12]}$ and elemental analysis) with respect to reaction time.

The isolated CN Raman line was identified as the most suitable feature for observing the distribution of the material through the bead. The long-
Table 1. Loading of the APS beads with 4-cyanobenzoic acid after different reaction times determined by ninhydrin assay and elemental analysis.

\begin{tabular}{ll}
\hline Reaction time $[\mathrm{min}]$ & Loading \\
\hline 0.5 & $29 \%$ \\
1 & $43 \%$ \\
2 & $56 \%$ \\
4 & $66 \%$ \\
6 & $75 \%$ \\
8 & $80 \%$ \\
20 & $88 \%$ \\
30 & $86 \%$ \\
40 & $91 \%$ \\
50 & $95 \%$ \\
\hline
\end{tabular}

term stability of the bead was investigated by leaving the bead under the laser for two hours and spectra taken every 12 minutes. There was no significant change in the intensity of the $1001 \mathrm{~cm}^{-1}$ polymer peak over this time indicating that there are no significant heating effects during the scans. The distribution of the $\mathrm{CN}$ species within the dry beads was initially analysed by scanning the beads along a line through the mid-plane of the bead in $1 \mu \mathrm{m}$ steps and recording a Raman spectrum at each point. Figure 1 shows the intensity of the Raman signal at $2230 \mathrm{~cm}^{-1}$ when plotted as a function of position through the bead with a loading of $29 \%$. An approximately linear relationship was observed between the CN Raman peak intensity and the loading (determined from the ninhydrin test) between 30-95\% loading.

Figure 1 clearly shows the sharp increase in relative intensity as soon as the edge of the bead is reached, while the intensity within the bead stays constant. The intensity near the edges of the bead was similar to that in the centre, in contrast to that implied by the observations of McAlpine et al. ${ }^{[8]}$ This holds true for all the samples analysed.

Figure 2 a) shows a map of a slice through the equatorial region of the bead. The different intensities of the $2230 \mathrm{~cm}^{-1}$ band are assigned to different shades of grey. Figure $2 \mathrm{~d}$ ) shows an example of a $\mathrm{CN}$ peak in the Raman spectrum and Figures $2 \mathrm{~b}$ ) and $2 \mathrm{c}$ show slices through the map in the $x$ and $y$ directions as indicated by the lines over the area map (Figure 2a)). Although only two examples are shown (Figures 2 3), all other spectra obtained from the differently
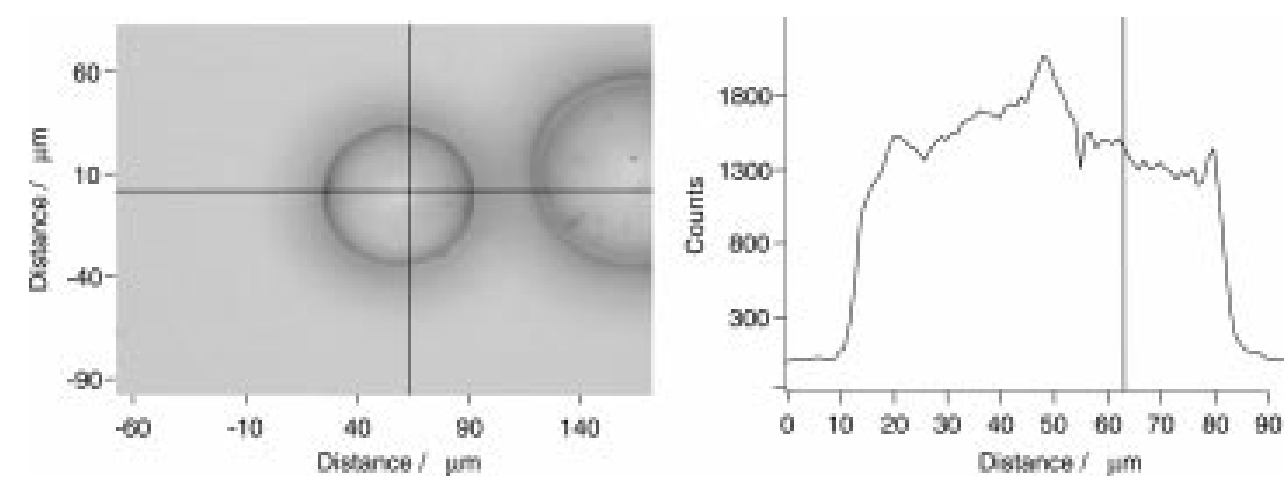

Figure 1. Raman intensity of the $2230 \mathrm{~cm}^{-1} \mathrm{CN}$ band of a dry $29 \%$ loaded APS bead along a horizontal scan through the equatorial plane of the bead. 


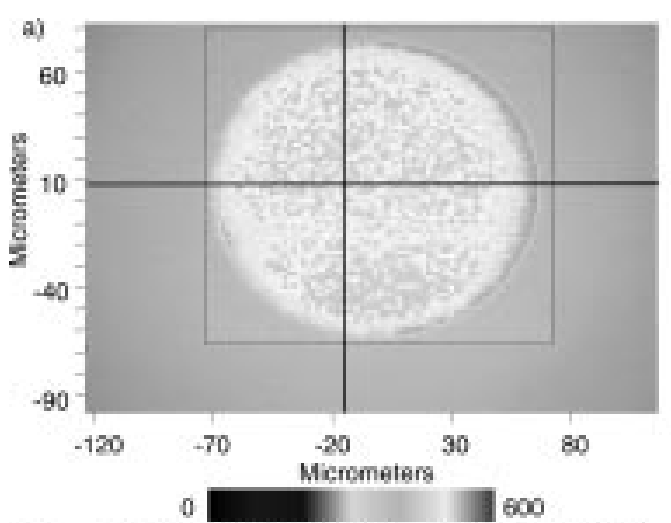

b) Data extracted along the $y$ axes at $x=-15.1517$
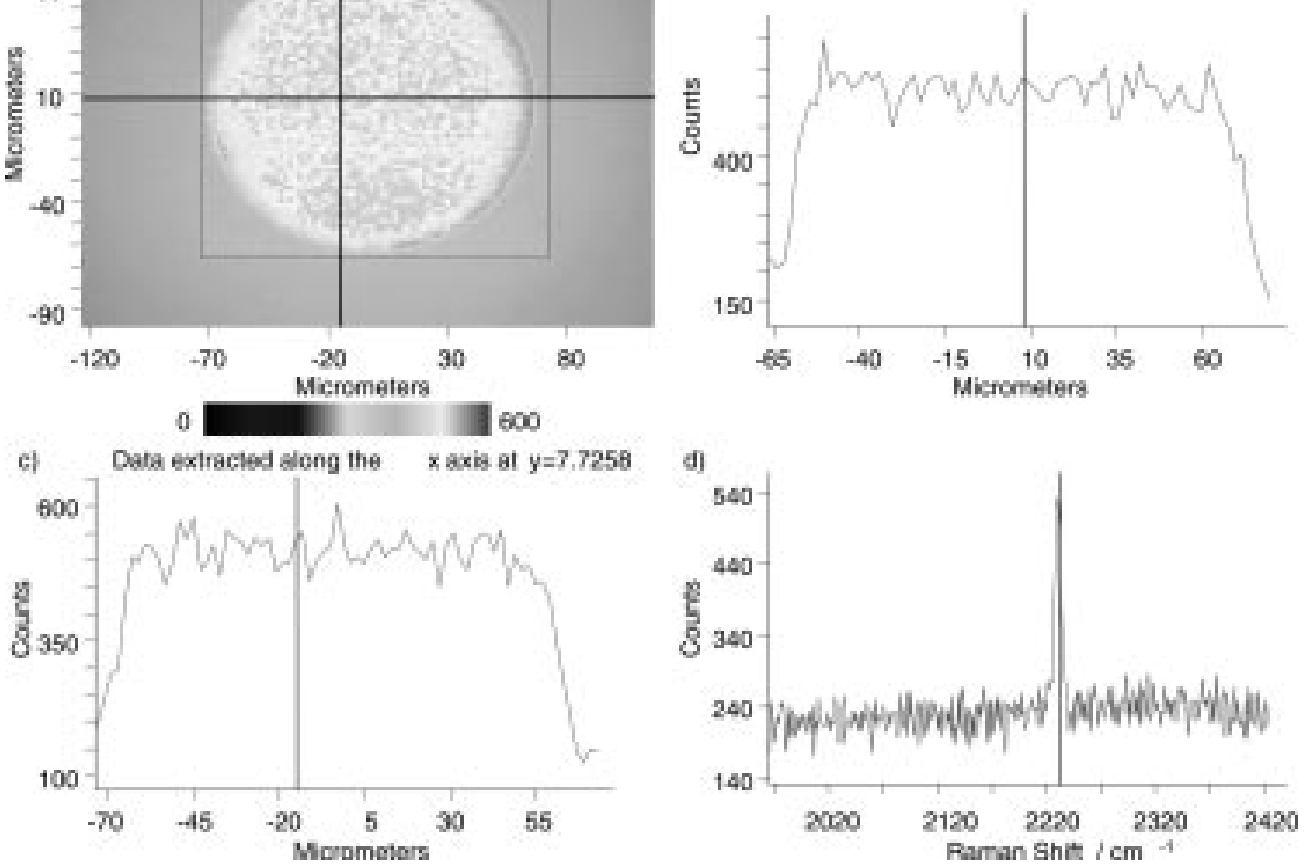

Figure 2. Raman intensity map of the $2230 \mathrm{~cm}^{-1} \mathrm{CN}$ band of a $95 \%$ loaded APS bead swollen in dioxane. The white light image of the bead is superimposed on the Raman map.
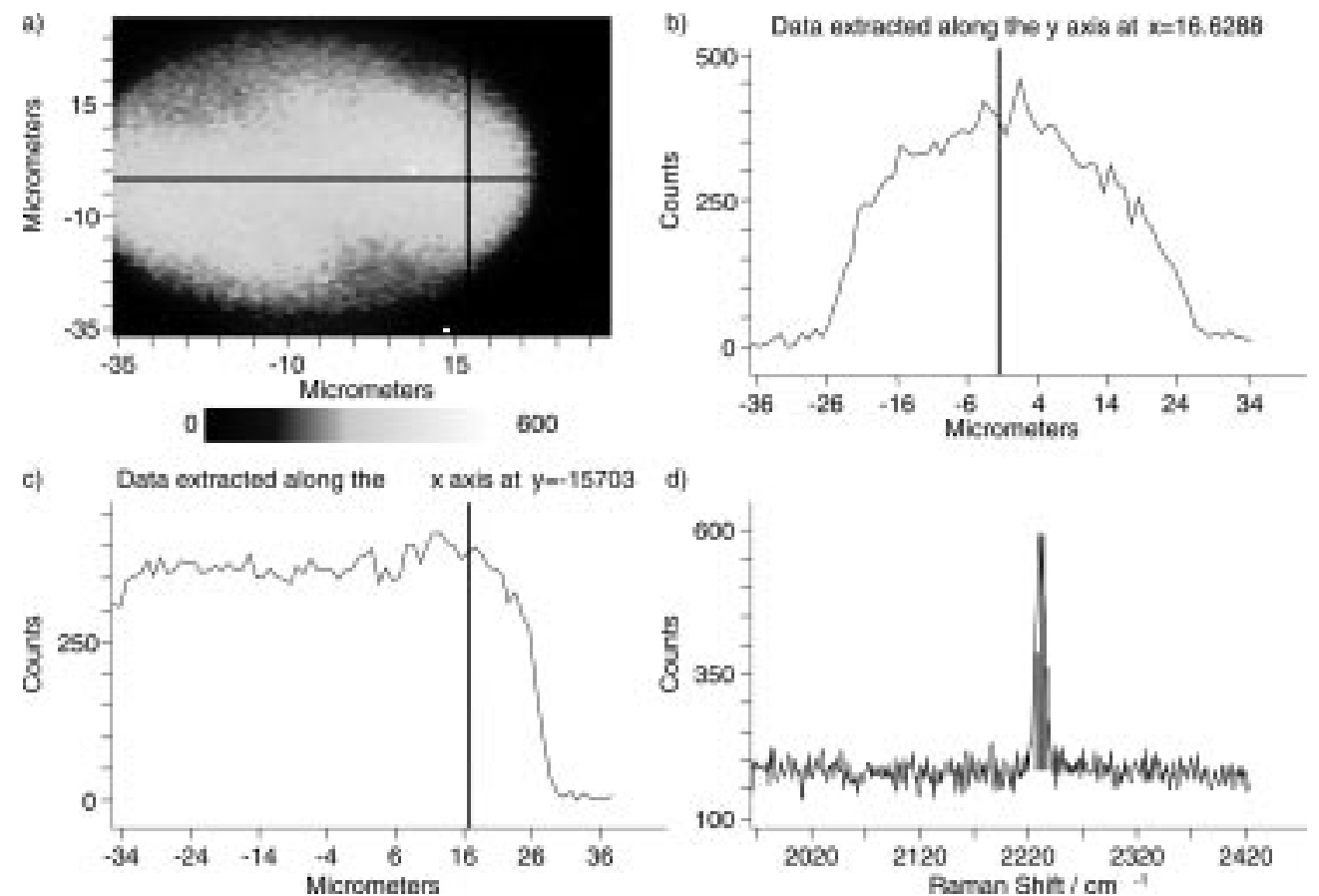

Figure 3. Raman intensity map of the $2230 \mathrm{~cm}^{-1} \mathrm{CN}$ and of a dry $95 \%$ loaded APS bead through the equatorial plane of the bead.

loaded beads (Table 1) showed the same features with respect to site distribution.

Similar experiments were carried out on dry beads. Examples of the resulting spectra are shown in Figure 3. Again there was no evidence for an unequal site distribution across the bead. The measurements were repeated on a variety of resin samples at different loadings, but all gave essentially the same results.
In summary, we have shown by the use of confocal Raman spectroscopy that the distribution of functional sites in aminomethylated polystyrene is uniform throughout the entire bead volume. We observed no "clusters" or segregation of the reactive sites. The use of the Raman technique (as opposed to a fluorescent technique) removes many of the possible ambiguities in the determination of the spatial distribution of the reactive sites. Furthermore our investiga- 
tion of dry and swollen beads is more relevant to the practical applications of these beads in solid-phase synthesis. Currently we are undertaking further studies on different bead materials including TentaGel, PEGA and controlled pore glass by confocal Raman and fluorescence microscopy.

\section{Experimental Section}

Aminomethylated polystyrene (NovaBiochem, $200-400$ mesh, $1 \%$ crosslinked, $0.04 \mathrm{~g}$ ) was placed in a filter funnel and swollen for $15 \mathrm{~min}$ in dioxane $(9.3 \mathrm{~mL})$. A solution of 4-cyanobenzoic acid $(0.023 \mathrm{~g}, 0.156 \mathrm{mmol})$ and 1,3-diisopropylcarbodiimide $(0.199 \mathrm{~g}, 0.156 \mathrm{mmol})$ in dioxane $(0.7 \mathrm{~mL})$ was prepared and added to the suspension of the resin. The reactions were quenched at different reaction times and thoroughly washed with the solvent. The resin was subsequently washed with $\mathrm{CH}_{2} \mathrm{Cl}_{2}, \mathrm{MeOH}$ and $\mathrm{Et}_{2} \mathrm{O}$ and finally dried in vacuo.

The resin beads were placed into the cavity of a microscopic slide, swollen in dioxane and covered with a $0.1 \mathrm{~mm}$ thick glass plate, which was fixed along the edges with three layers of parafilm, in order to prevent evaporation of the solvent during the measurement $(\approx 12 \mathrm{~h})$. An area of $148 \mu \mathrm{m} \times 148 \mu \mathrm{m}$ in the $x y$ plane through the equator of the bead was scanned in $2 \mu \mathrm{m}$ steps (giving a total of 5476 data points). Due to the confocal depth resolution this corresponds to approximately a $3 \mu \mathrm{m}$ slice through the bead. For the dry beads an area $70 \mu \mathrm{m} \times 70 \mu \mathrm{m}$ was scanned in $1 \mu \mathrm{m}$ steps (4900 data points).

In the mapping experiments a $50 \times$ objective was usually used, the slit set to $10 \mu \mathrm{m}$ on the spectrograph entrance assembly and the CCD area set to a four pixel height, in contrast to a $20 \mu \mathrm{m}$ slit width and a 20 pixel height for a non-confocal experiment. A silicon flat was used to determine the thickness of the confocal optical section. The measured depth profile gave a FWHM resolution of $3 \mu \mathrm{m}$. This results in a three-dimensional resolution of typically greater than about $1 \mu \mathrm{m} \times 1 \mu \mathrm{m} \times 3 \mu \mathrm{m}$ as the bead samples have a lower refractive index than the $\mathrm{Si}$ flat. For a line map the spectra were collected with between $2300 \mathrm{~cm}^{-1}$ and $900 \mathrm{~cm}^{-1}$ to allow both the peak of interest $\left(2230 \mathrm{~cm}^{-1}\right)$ and the reference polymer peak $\left(1001 \mathrm{~cm}^{-1}\right)$ to be observed. The area maps were recorded with a static scan with a range of $453 \mathrm{~cm}^{-1}$ (with the 1800 groove grating) centred at the peak of interest. The area to be mapped is drawn in software. The bead was focused manually on the bead centre and not with the auto-focus facility. The bi-directional scanning mode was used to avoid any jumps in the objective that may have caused the bead to move. For long scans ( $8 \mathrm{~h}$ plus)"cosmic ray removal" was turned on to remove large sporadic noise peaks disturbing the data. The spectra of the loaded beads were analysed using the GRAMS curve fitting application and the maps were created using the mapping program supplied with WiRE (Windows-based Raman environment) program from Renishaw.

\section{Acknowledgement}

We thank GW/EPSRC for Grant No. GR/M70797 funded through the combinatorial initiative.

[1] For recent reviews on the subject: a) K. S. Lam, M. Lebl, V. Krchnák, Chem. Rev. 1997, 97, 411-448; b) S. Booth, P. H. H. Hermkens, H. C. J.
Ottenheijm, D. C. Rees, Tetrahedron 1998, 54, 15385-15443; c) D. Hudson, J. Comb. Chem. 1999, 1,333-360; d) F. Guillier, D. Orain, M. Bradley, Chem. Rev. 2000, 100, 2091-2157; e) S. V. Ley, I. R. Baxendale, R. N. Bream, P. S. Jackson, A. G. Leach, D. A. Longbottom, M. Nesi, J. S. Scott, R. I. Storer, S. J. Taylor, J. Chem. Soc. Perkin Trans. 1 2000, 3815-4195.

[2] Polymer Handbook (Eds.: J. Brandrup, E. H. Immergut, E. A. Grulke), Wiley, New York, 1999.

[3] D. C. Sherrington, Chem. Commun. 1998, 2275-2286.

[4] V. K. Sarin, S. B. H. Kent, R. B. Merrifield, J. Am. Chem. Soc. 1980, $102,5463-5470$.

[5] S. B. Roscoe, J. M. J. Fréchet, J. F. Walzer, A. J. Dias, Science 1998, 280 , $270-273$.

[6] S. R. McAlpine, S. L. Schreiber, Chem. Eur. J. 1999, 5, 3528-3532.

[7] E. Bayer, Angew. Chem. 1991, 103, 117; E. Bayer, Angew. Chemie. Int. Ed. Engl. 1991, 30, 113-129.

[8] S. R. McAlpine, C. W. Lindsley, J. C. Hodges, D. M. Leonard, G. F. Filzen, J. Comb. Chem. 2001, 3, 1-5.

[9] a) B. J. Egner, S. Rana, H. Smith, N. Bouloc, J. G. Frey, W. S. Brocklesby, M. Bradley, Chem. Commun. 1997, 735-736; b) R. H. Scott, S. Balasubramanian, Bioorg. Med. Chem. Lett. 1997, $1567-$ 1572; c) B. Yan, P. C. Martin, J. Lee, J. Comb. Chem. 1999, 1, 78-81.

[10] B. R. Martens, Confocal Microscopy-Milestone Series, Vol. MS 131, Photo-Optical Instrumentation Engineers, 1996; G. J. Rosasco in Advances in Infrared and Raman Spectroscopy, Vol. 7 (Eds.: R. J. H. Clark, R. E. Hester), Wiley, Chichester, 1980.

[11] The confocal mode was used to obtain the highest spatial resolution. Since there is no significant absorption at $633 \mathrm{~nm}$ the illumination intensity will be the same throughout the bead. This contrasts with the potential difficulties with fluorescence spectroscopy mentioned above. The resolution of the confocal mode imaging in air is about $(1 \times 1 \times$ $3 \mu \mathrm{m})$ but previous work has shown that imaging within a material of higher refractive index can reduce the depth resolution by a factor of about $2{ }^{[13]}$ The influence of refraction at the curved surface of the bead must be included in the analysis of the Raman intensity. This is particularly true for the spectra recorded from the dry beads where the refractive index contrast between the bead and the surroundings is significant. The dry bead will act as a small spherical lens distorting the images taken through the beads. The images shown in Figure 3 have not been corrected for this distortion. However, for the swollen beads the refractive index is essentially identical to that of the surrounding solvent, since the beads contain such a high proportion of solvent. Under these circumstances there will be little or no refraction at the solvent/bead interface. Focussing the microscope into the solvent will give rise to some foreshortening due to refraction at the planar air/ solvent interface. This means that size of the bead as measured by the depth scan would be expected to be smaller than that given by the horizontal width scan by a factor of the refractive index of the swollen bead. For the dry beads (refractive index $\approx 1.6$ ) the focussing effect is significant, and gives rise to an asymmetric distortion of the vertical scans and a nonlinear but symmetrical distortion of the area scan. These distortions do not alter the general interpretation of the images of the dry beads and a detailed correction taking them into account is being prepared.

[12] V. S. Sarin, S. B. H. Kent, J. P. Tam, R. B. Merrifield, Anal. Biochem. 1981, 117, 147-157.

[13] N. J. Everall, Appl. Spectrosc. 2000, 54, 1515-1520.

Received: December 8, 2000 [F3193] 